This item was submitted to Loughborough's Research Repository by the author.

Items in Figshare are protected by copyright, with all rights reserved, unless otherwise indicated.

\title{
Sociocultural aspects of concussion
}

PLEASE CITE THE PUBLISHED VERSION

https://doi.org/10.4324/9781351200516

PUBLISHER

Routledge

VERSION

AM (Accepted Manuscript)

PUBLISHER STATEMENT

This is an Accepted Manuscript of a book chapter published by Routledge in Psychological Aspects of SportsRelated Concussions on 2019-03-13, available online: http://www.routledge.com/9780815391869

\section{LICENCE}

CC BY-NC-ND 4.0

\section{REPOSITORY RECORD}

Malcolm, Dominic. 2019. "Sociocultural Aspects of Concussion". Loughborough University. https://hdl.handle.net/2134/11871489.v1. 


\section{Sociocultural Aspects of Concussion}

6

7

Dominic Malcolm, Ph.D.

8

9

School of Sport, Exercise and Health Sciences

10

Loughborough University, UK

11

d.e.malcolm@lboro.ac.uk

12

ORCiD: 0000-0002-5898-2911

13

14

15

16

17

18

19

20

21

22

Version sent to GB+JC = July 24, 2018

23 
Table of Contents

26

27

28

29

30

31

32

33

34

35

36

37

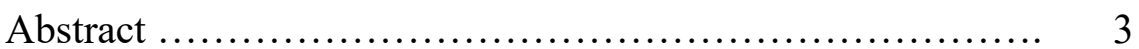

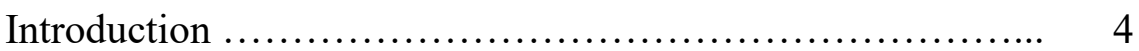

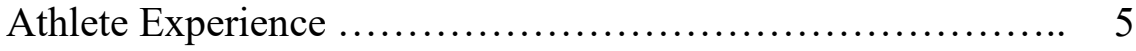

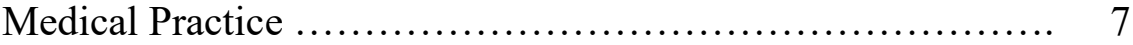

Medical Knowledge ..................................... 9

Public Health ............................................. 12

Cultural Representations .................................. 14

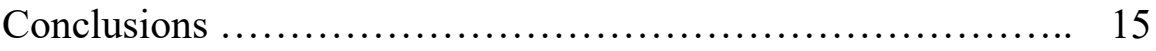

References ............................................. 18

Table ...................................................... 22 


\section{Abstract}

39 This chapter examines the various ways in which sociocultural analyses can enhance our

40 understanding of issues related to concussion. Identifying the emergence of an increasingly

41 multidisciplinary object of study, this chapter focuses on five areas of brain-injury related

42 sociocultural research — athlete experience; medical practice; medical knowledge; public

43 health; and cultural representations. It identifies how the peculiar social relations which form

44 sports cultures shape the attitudes and behaviors of athletes and clinicians who negotiate the

45 identification and management of concussion injuries, and how such social relations

46 influence the construction and dissemination of medical knowledge (in relation to

47 concussion). Additionally, this chapter explains how an awareness of sociocultural factors

48 can enhance our design and delivery of public health messages, and that effectively

49 understanding sport-related concussion requires a genuinely interdisciplinary field of study in

50 which sociocultural analysis plays an important part.

Keywords: concussion, interaction, social relations 


\section{Introduction}

Fundamental to a social scientific approach is an emphasis on sociocultural factors.

For sociologists of sport, concussion extends a well-established literature on subcultures of pain and injury in sport, including ideas about the relative tolerance of high levels of risk and violence that makes sport a unique sphere of social life (e.g., see Young, 2004). Concussion also resonates with the spectrum of issues that constitute the sociology of medicine, including: the analysis of patient experience and illness behaviors; lay-professional relations (including the relative influence of competing bodies of knowledge); the professional dominance of medicine; analysis of the social construction of disease via medical research and practice; health inequalities (e.g., in relation to gender or socioeconomic status); cultural representations of health-related conditions; and the political context of public health interventions (e.g., see Nettleton, 2006). But the unique parameters of concussion - the relatively limited scientific understanding, the uncertainty over longer term neurocognitive decline, the "vague and heterogenous" symptoms (McNamee, Partridge, \& Anderson, 2015, p. 193) - also make a sociocultural analysis particularly compelling.

However, it was only in the fifth and most recent Concussion in Sport Group (CISG) consensus statement (McCrory et al., 2017) that the significance of sociocultural factors in mediating experiences of mild traumatic brain injury was recognized. The statement explicitly stated that "Psychological and sociocultural factors in sport play a significant role in the uptake of any injury-prevention strategy and require consideration in terms of the existing limitations of injury prevention strategies" (McCrory et al., 2017, p. 8). In particular, through references to learning styles and preferred learning strategies of target audiences there appears to be a growing awareness that "knowledge transfer" requires a broader and multidisciplinary skill set. The consensus statement from McCrory and colleagues (2017) also recognized the degree to which psychological research had enhanced understanding of 
81 the factors influencing the recovery from concussion. Indeed, in identifying the significance

of factors such as sex, age, neurodevelopment, personal or family history of migraine, mental health problems, and past experiences of concussions there was a more implicit recognition of how concussion, as it is currently understood, needs to be considered within its broad sociocultural context.

This chapter develops this belated recognition and charts the various ways in which sociocultural analyses have and can enhance our understanding of issues related to concussion. It focuses on five areas of brain injury-related research: athlete experience; medical practice; medical knowledge; public health; and cultural representations. Although each is addressed here discretely for heuristic reasons, it should be noted that ultimately the promise of a sociocultural approach is to provide the kind of holistic and comprehensive analysis of concussion that can only be achieved by seeing the interdependence of these different areas.

\section{Athlete Experience}

Sociocultural analyses of athlete experiences of concussion first emerged in research focused on the cultures of risk in sport (Safai, 2003; Malcolm, 2009). This literature invokes ideas such as the "normalization" and "rationalization" of risk, pain, and injury as part of the “sport ethic" (Hughes \& Coakley, 1991), or “culture of risk” (Nixon, 1992). While studies identify considerable similarities in the way that male and female athletes respond, gendered identities have been highlighted as significant in the social construction of injury (Messner, 1992; White \& Young, 1999). In sum, athletes appear to talk about their bodies and understand injury in ways that are specific to sporting subcultures.

Sociologically, the key to understanding these forms of behavior is to investigate the distinct networks of relationships that characterize sport. While one of the first such studies from Safai (2003) identified a distinctly conservative orientation towards head injuries, this 
106 finding now appears to be the result of the approach espoused by a particularly influential

107 individual. More broadly, research suggests that brain injuries $d o$ evoke unique responses

108 from athletes, but that these tend to be characterized by less - rather than more - caution.

109 Specifically, in the precarious and insecure world of elite sports, where performance concerns

110 are invariably prioritized over health, athletes tend to be particularly reluctant to present their

111 symptoms of concussion to medical staff. Biomedical researchers (Broglio et al., 2010; Fraas,

112 Coughlan, Hart, \& McCarthy, 2013; McCrea, Hammeke, Olsen, Leo, \& Guskiewicz, 2004)

113 have identified three reasons for this, namely athletes': (a) perceptions that their condition is

114 not serious enough, (b) reluctance to leave the game and/or let down teammates, or (c)

115 disbelief that a concussion has occurred. Because athlete reluctance to consult with medical

116 staff is not unusual in sport (or even in the broader population), a sociocultural approach is

117 required to explain why the rationales presented for such behavior are particularly

118 pronounced in relation to concussion.

119 Malcolm's (2009) study of elite rugby explained how attitudes towards concussion

120 were shaped by the individual's perception that their trajectory of recovery was undetermined

121 and could have an unpredictable impact on their lives as athletes. Many reported that

122 concussions did not necessarily impair playing performance, but uncertainty over what might

123 happen if they revealed their concerns (would they, for instance, regain their place on the

124 team?) meant that rugby players were reluctant to withdraw from sporting activities.

125 Uncertainty also informed their decisions about whether to consult with medical staff. Players

126 knew that clinicians could not offer relief from symptoms or enable their recovery or return-

127 to-play except in the most severe cases. They also knew that alerting clinical staff to their

128 concerns about having suffered a concussion would likely only disrupt their careers and

129 identities through enforced withdrawal from sporting activities. In sum, while concussed

130 players may experience unusual bodily sensations, "they seldom experience problems that 
131 they themselves cannot resolve and rarely, or only briefly, experience uncertainty in the form

132 of concern about sporting performance” (Malcolm, 2009, p. 200).

134 documented athletes' distinctly risky behaviors in relation to sport-related concussions. Their

135 study of Irish amateur rugby players showed that individuals managed concussions by

136 downplaying, ignoring, denying the significance, or concealing symptoms and "playing on".

137 Post-injury consequences such as sleep disturbance, irritability, and mood swings were

138 largely hidden from teammates, coaches, etc. There was, moreover, testimony to the effect of

139 exhibiting a preference for receiving a concussion over musculoskeletal injuries. They argued

140 that the former frequently entailed a more limited impact on a person's ability to play rugby,

141 and even rationalized the experiences of concussion-induced cognitive impairment as

142 positive, "reverting" the individual to a "primal state" (deemed useful in the context of an

143 aggressive sport such as rugby). Severity of injury, conversely, was assessed by these players

144 as being directly related to the length of time one was unable to play sport, and excluded

145 considerations of longer term neurocognitive decline. This "head strong" attitude was derived

146 "from within the subculture of rugby, that is, originating in the level of commitment made by

147 players to each other and to the game" (Liston et al., in press, p. 9).

148 Consequently, sociocultural studies of athlete experiences enable us to see the

149 underlying logic to seemingly misguided or irrational behavior. The focus here is on the

150 interrelations between actors (athletes, coaches and clinicians) creating specific cultures of

151 risk in which a marked tolerance towards head injuries is a notable feature.

\section{Medical Practice}

A central premise of a sociocultural approach is that all healthcare treatment relies to

154 a significant degree on social interaction. While the previous section briefly referred to the

155 attitudes of athlete-patients towards clinicians, here the focus is on the impact of those 
156 interactions on the clinicians themselves. While essentially quantitative studies have

157 identified how non-compliance with concussion protocols is not unusual in professional sport

158 (Cusimano, Casey et al., 2017; Price, Malliaras, \& Hudson, 2012), a qualitative approach

159 provides: 1) a detailed illustration of the frequently stated assertion that clinicians find cases

160 of concussion particularly difficult to manage (McCrory et al., 2017); and perhaps more

161 significantly, 2) a demonstration of how clinicians' actions are not simply a product of their

162 training and professional instruction, but are fundamentally shaped through the interactions

163 they encounter as part of their role in patient management (Malcolm, 2009; 2017).

164 Comparable to the uncertainty athletes feel when their careers and identities are

165 threatened due to suspected concussion, healthcare professionals may experience uncertainty

166 through being unable to master all aspects of medical knowledge and practice. Fueling their

167 uncertainty in relation to concussion is the limited contemporary scientific knowledge base.

168 Consensus statements are replete with provisos and reservations about what we currently do

169 not know about concussion. For instance, it is notable how rarely, if at all, the position

170 statements produced by either the National Athletic Trainers' Association (Broglio et al.,

171 2014) or the American Medical Society for Sports Medicine (Harmon et al., 2013) provide

172 recommendations based on "consistent and good quality experimental evidence" (Broglio et

173 al., 2014, p. 246). Similarly, the 2017 CISG consensus statement notes that "critical questions

174 remain ... about the acute neurobiological effects of SRC on brain structure"; "insufficient

175 evidence" for prescribing complete rest; and currently "limited evidence to support the use of

176 pharmacotherapy" (McCrory et al., 2017, p. 4-5). Guidelines on how to manage concussion

177 have not only been repeatedly revised in recent years, but continue to defer to the primacy of

178 individual clinical judgement. Clinician interviewees reflect both their own uncertain

179 knowledge and the difficulties of working in a context where the guidance and instruction

180 they are given has frequently changed. For instance, they describe the difficulty of diagnosis, 
question the validity of existing protocols, and identify differences between sports as symptomatic of the lack of evidence (Malcolm, 2017).

The combination of uncertainty and skepticism linked to the changing regulatory environment generates three primary courses of action (Malcolm, 2009). First, clinicians may actively avoid the diagnosis of concussion. They may prefer to direct attention towards a different injury, or treat the head injury but simply resist formally labelling it as a concussion. They may instead emphasize that a player should be withdrawn as he would be unable protect himself in contact. Secondly, clinicians tend to stress that their experiential knowledge of the sport has enabled them to develop their own guidelines for practice which give primacy to particular signs and symptoms (Malcolm, 2009). Third, clinicians individualize treatment of their athletes, arguing that no two concussions are the same, and exerting their right to exercise clinical judgement over any textbook guidance or concussion identification tool. Prioritizing experiential knowledge and individualizing treatment essentially replaces a blanket "safety-first" approach with consideration of players' contributions to team performance. This effectively harmonizes the clinicians' approach with the concerns of players and coaches.

Ultimately sociocultural studies show the distinct difficulties in implementing diagnostic and treatment guidelines in high-pressured elite sports environments. Clinicians' accounts reveal practices which are frequently removed from the conservative philosophies of existing protocols but which are compatible with the primacy given to individual clinical judgement (Malcolm 2009; Malcolm 2017; see also Price, Malliaras, \& Hudson, 2012).

Clinical management problems essentially stem from the lack of strong evidential substance underpinning the relatively cautious recommendations. 
A sociocultural approach further embraces the idea that concussion, like all such conditions, relies on biomedical research and practice to identify, define, and subsequently delineate its parameters. Simply put, people have expressed concerns about brain injuries incurred through playing sport for many years (Harrison, 2014), yet it is the specific actions

209 of sporting and especially medical bodies that shape the contemporary understandings of the 210 mild traumatic brain injuries that have relatively recently come to define as concussion. One

211 can see this in the genesis of the CISG consensus statements where one of the major initial

212 concerns was to establish a definition of concussion around which researchers could focus

213 their endeavors. As such, this section will try to understand concussion through its social

214 construction in medical knowledge.

215 In "The medicalization of concussion", Malcolm (2017) argues that the sports

216 medicine community has defined concussion as an issue which does and should fall under the

217 provenance of medicine. The appointment of Paul McCrory as editor of the British Journal of

218 Sports Medicine was a significant catalyst in this. McCrory capitalized on this influential

219 position to rally, unify, and create a research agenda for a relatively disparate and divided set

220 of scholars in the first years of the $21^{\text {st }}$ Century. From these actions stemmed the formation of the CISG, the production of consensus statements, the expansion of research and, in particular, epidemiological studies. Epidemiology has certainly altered our understanding of the parameters of concussion as a social issue. Indicatively, epidemiology is a powerful tool

224 for raising public awareness of concussion, and this in turn may: 1) change people's

225 perceptions of certain bodily sensations they experience; and 2) provide them with a sociallylegitimate label through which to describe such sensations. The impact of this sociocultural critique goes beyond what was alleged to have been the deliberate and strategic attempts of the National Football League (NFL), through its Mild Traumatic Brain Injury Committee to have "fraudulently concealed" the risks of concussion and sub-concussive injuries to players 
230 (Hardes, 2017, p. 281). More simply, but no less systematically, through the identification of

231 key risk variables, contemporary epidemiology tends to emphasize individual responsibility

232 (e.g., poor tackling technique) and structural aspects of sports (e.g., the design of helmets,

233 rules which allow certain forms of contact), rather than subcultural norms of being "head

234 strong" that ultimately "cause" concussions. Articles written by the NFL's aforementioned

235 committee and controversially published in the journal Neurosurgery, were allegedly written

236 with a deliberate agenda to show that concussions were not a significant issue in the game

237 (Fainaru-Wada \& Fainaru, 2013).

238 A sociocultural analysis similarly questions the role consensus statements play in the

239 social construction of knowledge. As Bercovitz (2000) argues, consensus statements "may be

240 regarded as more a reflection of the desire of selected "experts" and scientists to impose their

241 world-view on research and practice" (p. 25). For example, Craton and Leslie (2014) have

242 contested the evidence basis for some of the conservative recommendations in the CISG

243 (2013) consensus statement, arguing that it: contains inclusion criteria for diagnosis which

244 are too broad and therefore could capture multiple other conditions; offers no supportive

245 evidence for prescribing physical and cognitive rest (now recognized as being a highly

246 problematic concept, anyway); and is reliant on the notion of "asymptomatic" to guide

247 graduated return to play, which is not operational, as most people are never fully

248 asymptomatic of the inclusion criteria (p. 93). The content of consensus statements should

249 thus be seen as a combination of medical "fact" and relatively subjective viewpoints

250 informed by the concerns expressed in broader public debates.

251 However, to fully understand the medicalization of concussion, we must go beyond

252 the actions of sports medicine personnel and explore the role of neuroscience (Hardes, 2017).

253 Concern about concussion has developed in tandem with a "brain culture" (Thornton, 2011),

254 where developments in knowledge, and particularly advances in imaging techniques, have 
offered the potential to provide a paradigmatic shift in our understanding of the human condition. However, neuroscience remains in its infancy and public faith in its potential vastly outstrips its current efficacy. This was evident in the successful legal action brought forth by NFL players, although the case settled in the absence of compelling evidence of a sport-concussion-Chronic Traumatic Encephalopathy (CTE) nexus.

In sum, a sociocultural approach is premised on a belief that scientific knowledge is not simply factual or neutral, but subject to human or social processes. The current state of concussion science should therefore be seen as the product of the attempts of particular researchers to establish a common core of understanding in a relatively contested and pioneering medical field.

\section{Public Health}

Sociocultural critiques of health promotion focus on three inter-related issues: structure, surveillance and consumption (Nettleton \& Bunton, 1995). Structurally, health promotion can be criticized for failing to recognize the importance of material (dis)advantage in mediating lifestyle and disregarding the significance of living conditions in contouring the choices that people can make. Typically, research in this area tends not to explore the heterogeneity in sporting populations' knowledge of concussion. Surveillance refers to the legitimacy of monitoring and regulating populations. This is especially complicated in relation to concussion as, by definition, the athlete-patient may not be able to make coherent decisions. But a sociocultural approach sensitizes us to the importance of recognizing that certain population cohorts actively seek out risk-taking behavior. Thus, while sociologists would agree that knowledge is often less important than the environment or relations in determining action (Kroshus, Daneshvar, Baugh, Nowinski, \& Cantu, 2014), they would ask further and fundamental questions about whether such messages were unequivocally in "the interests" of the target group whose relatively voluntary behaviors are 
being restricted. Finally, health promotion has been criticized for blurring the boundaries between medicine and consumer culture. Specifically in relation to concussion, the representation of sport-related brain injury has created a significant market for both preventative equipment (e.g., helmets) and concussion assessment tools which: 1) creates opportunities for the commercial exploitation of sports participants; and 2) constructs distinct and socially desirable lifestyles which are only available to those who are already relatively economically empowered. Consequently, consumptive elements of public health often perpetuate social structural inequalities.

A sociocultural perspective provides a more rounded understanding and therefore a more reliable basis on which to orientate injury prevention strategies. For instance, rather than seeing coaches as either benign or indeed a force for more conservative concussion management (see e.g., Sarmiento, Mitchko, Klein, \& Wong, 2010), these powerful individuals are viewed as a fundamental part of the problem. Similarly, a sociocultural perspective on public health highlights the dominance of psychological paradigms in health

294 promotion. Not only do these lead to an advocacy of individual responsibility for health

295 (Horrocks \& Johnson, 2014), and consequently facilitate victim-blaming and stigmatization, they draw attention away from the underlying social causes of behavior. Moreover, because sociocultural evidence suggests that health promotion aimed at individual behavioral change is most effective in relation to populations in favorable social and economic conditions

299 (Baum \& Fisher, 2014), we can better understand the uneven uptake of concussion injury

300 prevention programs. Currently surveys of such interventions generally "suggest short-term 301 improvements ... while the long-term gains are mixed" (Mrazik et al., 2015, p. 1552). Rather,

302 a sociocultural approach enables us to take a more holistic view of public health

303 interventions, raising fundamental questions about equality, the legitimacy of population

304 "control," and the potential for powerful commercial interests to exploit the opportunities that 
subsequently arise. By identifying how public health interventions replicate social structural inequalities, subject populations to high degrees of surveillance, and encourages them to engage in certain types of consumption, a sociocultural approach has the power to make injury prevention strategies more applicable and thus relevant to the lived experience of target populations.

\section{Cultural Representations}

Finally, it is important to consider the cultural representations of concussion on the Internet, in the print media, and via film and television. Major themes documented on social media include personal experiences of concussion, the reported incidence amongst professional athletes, dissemination of research and education, and policy and safety implications (Sullivan et al., 2012; Workewych et al., 2017). Surveys of online content have found that CTE and Alzheimer's disease, dementia or other cognitive problems are the most frequently cited consequences of concussion (Ahmed \& Hall, 2017). Analyses of print media have highlighted the focus on athletes' personal experiences. Anderson and Kian (2012) argued that concerns over head injuries have led to a softening of American sporting masculinity, while McGannon, Cunningham, and Schinke (2013) highlighted how the psychological implications of head injury are largely ignored. Finally, Furness's (2016) analysis of League of Denial highlights how the film challenges dominant sport media narratives in which representations of concussion normalize violence, injury, and hegemonic masculinity. Instead the film documents the institutional failings of the NFL which led to the landmark establishment of a $\$ 1$ billion compensation fund for former players.

More broadly, it can be argued that cultural representations of concussion have two primary consequences. First, the media tend to be relatively uncritical of the structural issues related to concussion. The consequence may be to underplay any broader political discussion of brain injury as a labor issue (Brayton et al., in press), or suggest that players are largely 
responsible for regulation failure. Some have argued that the media are therefore complicit in

331 the sustained "antipolitics" of the NFL's messages which hide a commodification of violence

332 within "the dangerous moral contours of racial capitalism" (Benson, 2017, p. 307). Second,

333 the media disseminates equivocal messages about the capacity of medicine to ameliorate

334 public concerns about concussion. While on the one hand, media coverage has been accused

335 of having "propagated an agenda of one-sided news and a sensationalised state of fear"

336 (Kuhn, Yengo-Kahn, Kerr, \& Zuckerman, 2017, p. 1732), other accounts have been critical

337 of the "overreliance on medico-scientific conceptions of CTE" (Ventresca, in press, p. 3)

338 ,which both ignore the current limitations in scientific knowledge and in so doing exaggerate

339 the potential of biomedicine to resolve both individual experiences and broader public 340 concerns over sporting concussions (Malcolm, 2017).

A vital contribution of a sociocultural approach, therefore, is to locate athlete and

342 clinician experiences, medical research and health promotion, within their cultural context.

343 Analysis of the cultural representation of concussion enables us to consider concussion not

344 just as a unique type of sports injury, but as a condition which the population understands in

345 ways that are structured according to broader social, political, and economic interests.

\section{Conclusions}

Building on what has only been a relatively recent recognition that sociocultural

factors are significant in mediating the experience and management of concussion (McCrory et al., 2017), this chapter has sought to provide an overview of how concussion must be conceived of as impacting across a range of social domains - from cultural representations to public health, medical knowledge, medical practice and athlete experience. Specifically, this chapter has identified how the peculiar social relations which form sports cultures shape the attitudes and behaviors of both the athletes and clinicians who negotiate the identification and

354 management of concussion injuries. It has further examined how such social relations 
influence the construction and dissemination of medical knowledge (in relation to concussion) and how an awareness of sociocultural factors can enhance our design and delivery of public health messages designed to reduce the incidence and health costs of this particular sports injury. Noting how the populations' understanding of concussion is literally mediated through broader narratives of cultural representation reminds us of the interconnectedness of these apparently diverse social domains. Public perceptions, clinicians' actions, and even medical consensus statements do not develop in a vacuum. This point underscores the ultimate promise of a sociocultural approach; to provide a more comprehensive and systematic understanding of what is clearly a complex medical and social problem.

If the science of concussion can be said to still be in its infancy (McCrory et al., 2017), then sociocultural analyses of this medical and social phenomenon have barely been born. Consequently, there is a clear need to identify what research we need and how it might further our understanding of concussion. Complicating the establishment of this agenda is the existence of multiple disciplinary voices all speaking to the issue of concussion and making their pitch to be heard. As I have attempted to show here, sociocultural research should remain true its disciplinary principles, and critical sociological researchers should not simply want to support or bolster the work of medicine (although clearly that is both worthwhile and will lead to important social benefits), but must also reflect upon and evaluate the concept and social functioning of health per se. This is what I attempt to illustrate in the concluding paragraphs.

In summarizing the breadth of potential sociocultural contributions to an understanding of concussion (see Table 1), it may be helpful to cite Stefan Timmermans' (2013) seven views (or warrants) about the unique contributions of qualitative health sociology, and how this might be manifest in concussion research (Malcolm, in press). 
380 Timmermans is a leading sociologist of health and illness scholar and the Senior Medical

381 Sociology editor for the journal Social Science and Medicine. The first column of Table 1

382 reports each of Timmermans' seven warrants, and the second column describes how I

383 imagine these might be applied to sociocultural research on sports concussion.

[Insert Table 1 here]

For many more familiar with the natural sciences, this array of aims and objectives may seem dauntingly broad, if not naively over-ambitious. But for those committed to a sociocultural analysis they provide an antidote to the sometimes narrow and overly individualistic, patient- or bodily-focused approach of biomedicine. Where sports medicine literature describes concussion as a particularly complex condition, a sociocultural approach explains how the network of interdependencies makes this so. Ultimately it must be recognized that the social sciences have something different-advocates might say uniquely beneficial - to offer our understanding of concussion. It is both my contention that concussion, for various reasons (it seems to be an urgent social issue; it seems to be beyond

394 simple or quick fixes; it seems that the social context in which it occurs places considerable

395 strain on biomedical practices) is distinctively placed to become a genuinely interdisciplinary

396 field of study. It is my belief that this will benefit the many millions of people who sustain

397 concussions each year, and ensure that sport remains broadly popular and continues to confer

398 distinct health benefits for the population. It is also my hope that that will occur. Now that the

399 importance of the contribution of sociocultural approaches has been recognized by

400 biomedical researchers (McCrory et al., 2017), it is vital that those who are fully conversant 401 with such approaches actively begin the shape the concussion research agenda. 


\section{References}

Ahmed, O., \& Hall, E. (2017). "It was only a mild concussion": Exploring the description of sports concussion in online news articles. Physical Therapy in Sport, 23, 7-13.

Anderson, E., \& Kian, E. (2012). Examining media contestation of masculinity and head trauma in the National Football League. Men \& Masculinities, 15, 152-173.

Baum, F. \& Fisher, M. (2014). Why behavioural health promotion endures despite its failure to reduce health inequalities. Sociology of Health and Illness, 36, 213-225.

Benson, P. (2017). Big football: Corporate social responsibility and the culture and color of injury in America's most popular sport. Journal of Sport \& Social Issues, 41, 307-334.

Bercovitz, K. (2000) A critical analysis of Canada's “Active Living”: Science or politics? Critical Public Health, 10, 19-39.

Brayton, S., Helstein, M., Ramsey, M. \& Rickards, N. (in press). Exploring the missing link between the concussion "crisis" and labor politics in professional sports. Communication and Sport.

Broglio, S., Cantu, R., Gioa, G. et al. (2014). National Athletic Trainers' Association position statement: Management of sport concussion. Journal of Athletic Training, 49, 245-265.

Broglio, S., Vagnozzi, R., Sabin, M., Signoretti, S., Tavazzi, B., \& Lazzarino, G. (2010) Concussion occurrence and knowledge in Italian football (soccer), Journal of Sports Science and Medicine, 9, 418-430.

Craton, N. \& Leslie, O. (2014). Time to re-think the Zurich guidelines? A critique on the consensus statement on concussion in sport, held in Zurich, November 2012. Clinical Journal of Sports Medicine, 24, 93-95.

Cusimano, M., Casey, J., Jing, R., Mishra, A., Solarski, M., Techar, K., \& Zhang, S. (2017). Assessment of head collision events during the 2014 FIFA World Cup tournament. Journal of the American Medical Association, 317, 2548-2549.

Fainaru-Wada, M., \& Fainaru, S. (2013). League of denial. New York: Crown Business.

Fraas, M., Coughlan, G., Hart, E. \& McCarthy, C. (2013). Concussion history and reporting rates in elite Irish rugby union players. Physical Therapy in Sport, 15, 136-142.

Furness, J. (2016). Reframing concussions, masculinity, and NFL mythology in League of Denial. Popular Communication, 14, 49-57.

Hardes, J. (2017). Governing sporting brains: Concussion, neuroscience, and the biopolitical regulation of sport. Sport, Ethics \& Philosophy, 11, 281-293. 
Harmon, K., Drezner, J., Gammons, M., Guskiewicz, K., Halstead, M., Herring, S. A., ... Roberts, W. O. (2013). American medical Society for Sports Medicine position statement: Concussion in sport. British Journal of Sports Medicine, 47, 15-26.

Harrison, E. (2014). The first concussion crisis: Head injury and evidence in early American football, American Journal of Public Health, 104, 822-833.

Horrocks, C. \& Johnson, S. (2014). A socially situated approach to inform health and wellbeing. Sociology of Health and Illness, 36, 175-186.

Hughes, R. \& Coakley, J. (1991). Positive deviance among athletes: The implications of the sport ethic. Sociology of Sport Journal, 8, 307-25.

Kroshus, E., Daneshvar, D., Baugh, C., Nowinski, C. \& Cantu, R. (2014). NCAA concussion education in ice hockey: An effective mandate. British Journal of Sports Medicine, 48, 135-140.

Kuhn, A., Yengo-Kahn, A., Kerr, Z., \& Zuckerman, S. (2017). Sports concussion research, chronic traumatic encephalopathy and the media: Repairing the disconnect. British Journal of Sports Medicine, 51, 1732-1733.

Liston, K., McDowell, M., Malcolm, D., Scott, A. \& Waddington, I. (in press). On being "Head Strong": The pain zone and concussion in non-elite rugby union. International Review for the Sociology of Sport.

Lupton D. (1995). The imperative of health. Public health and the regulated body. London: Sage.

Malcolm, D. (2009). Medical uncertainty and clinician-athlete relations: The management of concussion injuries in rugby union. Sociology of Sport Journal, 26, 191-210.

Malcolm, D. (2017). Sport, medicine and health: The medicalization of sport? London: Routledge.

Malcolm, D. (in press). Concussion in sport: Public, professional and critical sociologies, Sociology of Sport Journal.

McCrea, M., Hammeke, T., Olsen, G. Leo, P., \& Guskiewicz, K. (2004). Unreported concussion in high school football players: Implications for prevention. Clinical Journal of Sports Medicine, 14, 13-17.

McCrory, P., Meeuwisse, W., Dvorak, J., Aubry, M., Bailes, J., Broglio, S., ... Davis, G. A. (2017) Consensus statement on concussion in sport - the 5th International Conference on Concussion in Sport held in Berlin, October 2016. British Journal of Sports Medicine, 51, 838-847.

McGannon, K., Cunningham, S., \& Schinke, R. (2013). Understanding concussion in sociocultural context: A media analysis of a National Hockey League star's concussion. Psychology of Sport and Exercise, 14, 891-899. 
McNamee, M., Partridge, B. \& Anderson, L. (2015). Concussion in sport: Conceptual and ethical issues. Kinesiology Review, 4, 190-202

Messner, M. (1992). Power at play: Sports and the problem of masculinity. Boston, MA: Beacon Press

Mrazik, M., Dennison, C., Brooks, B. Yeates, K. Babul, S. \& Naidu, D. (2015) A qualitative review of sports concussion education: Prime time for evidence-based knowledge translation. British Journal of Sports Medicine, 49, 1548-1553.

Nettleton, S. (2006). The Sociology of Health and Illness (2 $2^{\text {nd }}$ edition). Cambridge: Polity.

Nettleton, S. \& Bunton, R. (1995). Sociological critiques of health promotion. In R. Bunton, S. Nettleton \& R. Burrows (Eds.), The sociology of health promotion: Critical analyses of consumption, lifestyle and risk (pp. 39-56). London: Routledge.

Nixon, H.L. II (1992). A social network analysis of influences on athletes to play with pain and injuries. Journal of Sport and Social Issues, 16, 127-35.

Povvidenza, C., Engebretsen, L., Tator, C., Kissick, J., McCrory, P., Sills, A., \& Johnston, K. (2013) From consensus to action: knowledge transfer, education and influencing policy on sports concussion. British Journal of Sports Medicine, 47, 332-338.

Price, J., Malliaras, P. \& Hudson, Z. (2012). Current practices in determining return to play following head injury in professional football in the UK. British Journal of Sports Medicine, 46, 1000-1003. doi:10.1136/bjsports-2011-090687

Safai, P. (2003). Healing the body in the "culture of risk": Examining the negotiations of treatment between sport medicine clinicians and injured athletes in Canadian intercollegiate sport. Sociology of Sport Journal, 20, 127-46.

Sarmiento, K., Mitchko, J. Klein, C \& Wong, S. (2010). Evaluation of the Centers for Disease Control and Prevention's concussion initiative for high school coaches: "Heads up: Concussion in High School Sports”. Journal of School Health, 80, 112-118.

Sullivan, S., Schneiders, A., Cheang, C., Kitto, E., Lee, H., Redhead, J., ... McCrory, P. (2012). "What's happening?" A content analysis of concussion-related traffic on Twitter. British Journal of Sports Medicine, 46, 258-263.

Thornton, D.J. (2011). Brain culture: Neuroscience and popular media. London: Rutgers University Press.

Timmermans, S. (2013). Seven warrants for qualitative health sociology. Social Science and Medicine, 77, 1-8.

Ventresca, M. (in press). The curious case of CTE: mediating materialities of traumatic brain injury. Communication and Sport.

White, P. \& Young, K. (1999). Sport and gender in Canada, Don Mills, ON: Oxford University Press 
Williams, J.M., Langdon, J.L., McMillan, J.L., \& Buckley, T.A. (2016). English professional football players' concussion knowledge and attitude. Journal of Sport Health Science, 6, 197-204.

Workewych, A., Muzzi, M., Jing, R., Zhang, S., Topolovec-Vranic, J., \& Cusimano, M. (2017). Twitter and traumatic brain injury: A content and sentiment analysis of tweets pertaining to sport-related brain injury. SAGE Open Medicine, 5, 1-11.

Young, K. (2004). Sporting bodies, damaged selves: Sociological studies of sport-related injury. Oxford: Elsevier. 
A research agenda for sociocultural research on concussion

\begin{tabular}{|c|c|}
\hline $\begin{array}{l}\text { Warrant of health sociology } \\
\text { (Timmermans, 2013) }\end{array}$ & Application to sports concussion research \\
\hline $\begin{array}{l}\text { Explore the social } \\
\text { construction of beliefs }\end{array}$ & $\begin{array}{l}\text { Illustrate the processes by which the diagnosis and } \\
\text { management of concussion are shaped by the distinct } \\
\text { characteristics of sport as a setting for medical practice }\end{array}$ \\
\hline $\begin{array}{l}\text { Reframe dominant } \\
\text { perspectives }\end{array}$ & $\begin{array}{l}\text { Question existing ideas that the 'solution' to sport's } \\
\text { 'concussion crisis' lies solely or predominantly within } \\
\text { the medical domain, and identify the potential } \\
\text { contributions that might be made by alternative } \\
\text { paradigms or ways of thinking }\end{array}$ \\
\hline $\begin{array}{l}\text { Chart the winners and losers } \\
\text { of health interventions }\end{array}$ & $\begin{array}{l}\text { Identify the existence and resultant impact of } \\
\text { inequalities in medical provision between population } \\
\text { cohorts, drawing attention to the way in which the } \\
\text { medicalization of concussion may lead to aspects of } \\
\text { social exclusion or social stratification of exercising } \\
\text { populations }\end{array}$ \\
\hline $\begin{array}{l}\text { Identify the unfulfilled } \\
\text { promises of medicine }\end{array}$ & $\begin{array}{l}\text { Scrutinize the degree to which the claims made by } \\
\text { medicine to be able to resolve concussion issues are } \\
\text { evident in practice, and the degree to which existing } \\
\text { practice is consistent with fundamental medical ethical } \\
\text { promises such as 'first do no harm' }\end{array}$ \\
\hline $\begin{array}{l}\text { Explore the experiences of } \\
\text { medicine and health across } \\
\text { multiple contexts }\end{array}$ & $\begin{array}{l}\text { Illustrate cross-sport, cross-gender and cross-cultural } \\
\text { similarities and differences in concussion attitudes and } \\
\text { behavior to reveal the spectrum of human experience, } \\
\text { and those practices which are distinct to, and therefore } \\
\text { a product of, contemporary Euro-American contexts }\end{array}$ \\
\hline $\begin{array}{l}\text { Expose the impact of } \\
\text { economic interests }\end{array}$ & $\begin{array}{l}\text { Reveal which groups economically profit from the } \\
\text { ever-increasing opportunities that arise from the threats } \\
\text { posed by concussion injuries to both individuals and } \\
\text { particular sports }\end{array}$ \\
\hline $\begin{array}{l}\text { Reveal how social relations } \\
\text { mediate the impact of } \\
\text { medical and health } \\
\text { interventions }\end{array}$ & $\begin{array}{l}\text { Provide a better understanding of how the social } \\
\text { relations entailed in consultations about, and the } \\
\text { diagnosis and management of, concussion can } \\
\text { contribute fundamentally to the wellbeing of human } \\
\text { populations }\end{array}$ \\
\hline
\end{tabular}

